
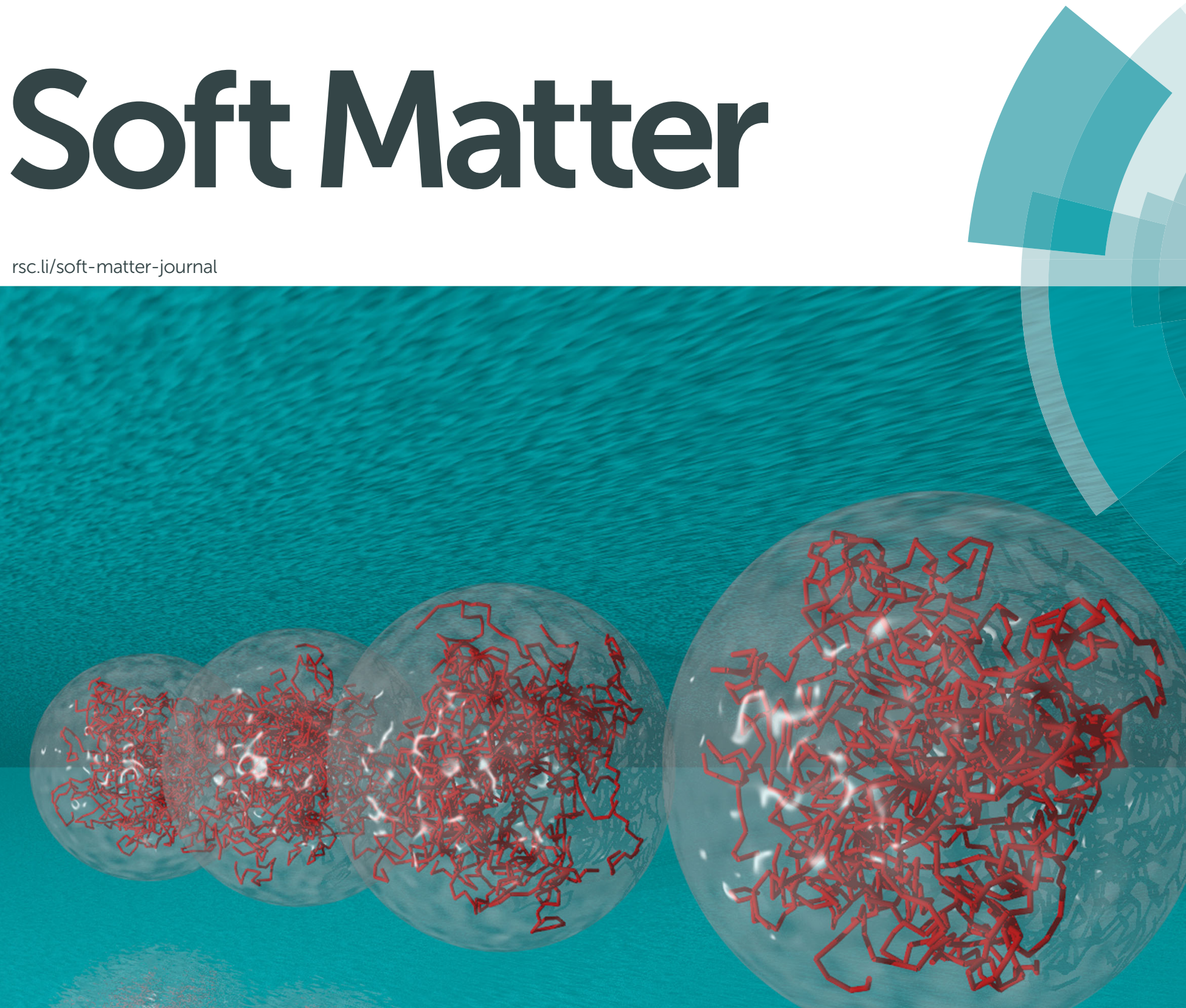

ISSN 1744-6848 
Check for updates

Cite this: Soft Matter, 2019, 15, 9614

Received 6th September 2019, Accepted 29th September 2019

DOI: 10.1039/c9sm01802f

rsc.li/soft-matter-journal

\title{
Microgels as viscosity modifiers influence lubrication performance of continuum $\dagger$
}

\author{
Efren Andablo-Reyes, ${ }^{a}$ Demetra Yerani, ${ }^{a}$ Ming Fu, ${ }^{a}$ Evangelos Liamas, (D) ${ }^{a}$ \\ Simon Connell, ${ }^{b}$ Ophelie Torres ${ }^{a}$ and Anwesha Sarkar (D)*a
}

\begin{abstract}
Biocompatible microgels have been demonstrated to act as excellent lubricants, however, the influence of the continuum on their overall mechanical performance has been neglected so far. In this work, the mechanical performance of colloidal whey protein microgels (hydrodynamic diameter $\sim 100 \mathrm{~nm}$ measured using dynamic light scattering and atomic force microscopy) of different rigidity dispersed in Newtonian (buffer and corn syrup) or complex non-Newtonian fluids (xanthan gum) is investigated for the first time via rheology and soft tribology. Dispersions of both soft microgels $\left(G^{\prime} \sim 100.0 \mathrm{~Pa}\right)$ and hard microgels $\left(G^{\prime} \sim 10.0 \mathrm{kPa}\right.$ ) were observed to act as thickeners in buffer as well as in low viscosity corn syrup and correspondingly reduced the friction, latter decreased as a function of the increased rigidity of the microgels. Differently, in high viscosity continuum, the microgels acted as thinning agents and increased the friction. In the lubrication limit, microgels in buffer or corn syrup behaved as Newtonian fluids with effective viscosity corresponding to their second Newtonian plateau value $\left(\eta_{\infty}\right)$. However, the lubrication performance of the microgels dispersed in the complex fluid (xanthan gum) could not be described quantitatively by $\eta_{\infty}$. For the low viscosity xanthan gum, the microgels had no influence on friction. Nevertheless, for the high viscosity counterparts, the soft microgels acted as thinning agents whilst the hard microgels accelerated the onset of elastohydrodynamic regime. This study demonstrates that microgels act as viscosity modifiers directly influencing the tribological performance, depending upon a subtle interplay of rheological properties of the particles and continuum.
\end{abstract}

\section{Introduction}

Microgels are soft colloids made of cross-linked polymers that are capable of entrapping significant proportions of solvent or water. Their composition and size, which ranges from tens of nanometers to hundreds of microns, can be tailored in order to provide desirable chemical and physical characteristics to target different applications. ${ }^{1}$ Recently, biocompatible microgels, such as those made up of proteins have shown remarkable properties in highly demanding biological applications, such as drug delivery, ${ }^{2,3}$ altering interfacial digestion kinetics ${ }^{4}$ and lubrication of biological contacts. ${ }^{5}$ For instance, submicronsized whey protein microgels have been used to stabilise Pickering emulsion, providing a transient barrier against enzymatic diffusion by lipase. ${ }^{6}$ Another example of microgel performance is lubrication, which is essential in the development of physiological processes involved in mechanical contacts, such as highly loaded synovial joints as well as delicate rubbing

\footnotetext{
${ }^{a}$ Food Colloids and Bioprocessing Group, School of Food Science and Nutrition, University of Leeds, UK. E-mail: A.Sarkar@leeds.ac.uk

${ }^{b}$ Molecular and Nanoscale Physics Group, School of Physics and Astronomy, University of Leeds, UK

$\dagger$ Electronic supplementary information (ESI) available. See DOI: 10.1039/c9sm01802f
}

surfaces like eyelids. ${ }^{7}$ Sensory properties related to skin or oral processing are also examples where lubrication is of paramount importance. For instance, recent studies have demonstrated correlation between the tribological performance of edible hydrogels in soft silicon contacts with oral perception attributes, such as pastiness and slipperiness ${ }^{8}$ and effects of such unique lubrication on satiety responses. ${ }^{9}$

Aqueous lubrication has been recognized as one of the main mechanisms for bio-lubrication due to the abundance of water in complex living organisms. ${ }^{10}$ Aqueous lubrication is attributed to the capacity of biopolymers to electrostatically bind water molecules while being tethered to the bio-surfaces. Hydrated layers formed near the surfaces by this mechanism are capable of supporting high pressure such as those developed in synovial joints. ${ }^{11}$ The aqueous lubrication performance of colloidal microgels has recently attracted research attention, such as in the case of particles made of thermo-responsive hydrogels $^{12}$ or biopolymers including starch, ${ }^{13}$ whey protein ${ }^{14,15}$ as well as non-starch polysaccharides (alginate, ${ }^{16}$ agarose,$^{17}$ $\kappa$-carrageenan $\left.{ }^{18}\right)$. The capacity of these soft particles to reduce friction in soft mechanical contacts has been attributed to their action as surface separators in combination to a roll bearing mechanism, the latter phenomenon is still under debate. ${ }^{13}$ 
An important aspect which has been as-of-yet neglected in the literature, whilst possibly having an exceptional relevance in the mechanical performance of the colloidal dispersions, is the role of the continuous phase i.e., the continuum in which the microgels are dispersed. ${ }^{19}$ Farres et al. carried out an elegant study where the role of co-solutes, such as glucose and glycerol on the lubrication properties of agar fluid gels was investigated. $^{20}$ The authors proposed that altering both the rate of ordering transition and the degree of solvation of the gel particles by the co-solutes present in the continuum could influence the lubricating behaviour of fluid gels. Nevertheless, many, if not most studies conducted on microgel or fluid gel lubrication have focused on simple aqueous dispersions. Complex fluids are, in general terms, a combination of a variety of microscopic components with different microstructures, such as colloidal particles and polymers. The self and collective dynamics and configuration of these components contribute in different manner to the macroscopic behaviour of the material. ${ }^{21}$ For instance, the response of complex fluids to mechanical deformation is dominated by a wide spectrum of relaxation times associated with different levels of component level reorganisation, such as chain segments decorrelation ${ }^{22}$ or colloidal mesostructures breakage under shear. ${ }^{23}$ The understanding of the mechanical behaviour of microgel dispersions in complex continuum might also be relevant as simple aqueous media are often altered with the addition of rheology modifiers in real-life applications. Particularly such fundamental knowledge will open up possibilities for templating new soft microgel + complex continuum combinations with optimized performance, such as shear-thinning with ultralow friction coefficients, and will pave the way towards the development of novel functional biomedical, personal care and nutritional applications.

In this work, colloidal whey protein particles are investigated as models of soft microgels dispersed in continuums of different rheological behaviours. Whey protein microgels have been recently regarded as important biocompatible lubricants. ${ }^{14,15}$ Two different types of well-characterised fluids are used as continuum in this study. On the one hand, corn syrup solutions with different viscosities are used as Newtonian continuum to serve as a comparison with various literature. On the other hand, xanthan gum solutions are investigated as examples of complex non-Newtonian continuum. While the lubrication performance of corn syrup has been found to be controlled by the viscosity ${ }^{24}$ of the Newtonian fluid, the lubrication capacity of xanthan gum has also been largely attributed to aqueous lubrication. ${ }^{25,26}$ In addition to the changes in the continuum properties, the role of the particle softness was also investigated by synthesising microgels of different whey protein content to influence the corresponding protein-crosslinking densities. Variation of mechanical characteristics of microgels and continuum allowed establishing a relationship between the high shear rheology of the dispersions and their lubrication properties, which to the best of our knowledge has never been reported for this kind of complex continuum before.

\section{Experimental}

\subsection{Materials}

Whey protein isolate (WPI) powder containing $96.3 \mathrm{wt} \%$ protein was kindly donated by Fonterra Limited (Auckland, New-Zealand). Xanthan gum was purchased from Sigma Aldrich, Dorset, UK and used as received. Corn syrup was purchased from Special Ingredients Ltd (Chesterfield, UK). Monosodium phosphate $\left(\mathrm{NaH}_{2} \mathrm{PO}_{4}\right)$ and disodium phosphate $\left(\mathrm{Na}_{2} \mathrm{HPO}_{4}\right)$ were purchased from VWR Chemicals, UK. Milli-Q water (resistivity of $18 \mathrm{M} \Omega \mathrm{cm}$ by Milli-Q apparatus, Millipore Corp., Bedford, MA, USA) was used for preparation of $20 \mathrm{mM}$ phosphate buffer at $\mathrm{pH}$ 7.0.

\subsection{Methods}

Preparation of whey protein gels. Whey protein solution was prepared by dissolving exact quantities of whey protein isolate (10.0 wt\% or $15.0 \mathrm{wt} \%$ ) in $20 \mathrm{mM}$ phosphate buffer at $\mathrm{pH} 7.0$ and stirred for 2 hours at room temperature to ensure complete dissolution. The disulphide crosslinking of whey protein was achieved by heating the whey protein solutions at $90{ }^{\circ} \mathrm{C}$ for 30 minutes. Thermally-crosslinked whey protein gels were allowed to cool down at room temperature before placing them at $4{ }^{\circ} \mathrm{C}$ for 12 hours. Although both concentrations of protein produce soft deformable gels, in this study, $10.0 \mathrm{wt} \%$ is referred as soft gels whilst $15.0 \mathrm{wt} \%$ is referred as hard gel.

Preparation of whey protein microgel (WPM) dispersions in Newtonian continuum. Phosphate buffer or two different concentrations of corn syrup solutions, $50.0 \mathrm{wt} \%$ and $75.0 \mathrm{wt} \%$, were used as the Newtonian continuum for the preparation of microgel dispersions. Solutions were prepared by mixing corn syrup with phosphate buffer at room temperature and stirred at $40{ }^{\circ} \mathrm{C}$ for an hour until a homogeneous solution was obtained.

The previously crosslinked whey protein gels (10.0 wt $\%$ or $15.0 \mathrm{wt} \%$ ) were mixed with either phosphate buffer or corn syrup solutions at a $1: 1 \mathrm{w} / \mathrm{w}$ ratio and subsequently sheared with a hand blender (HB711M, Kenwood, UK) for 1 minute. The whey protein gel dispersed in either the phosphate buffer or the corn syrup was then placed under vacuum for 30 minutes in order to remove the excess air introduced during blending. The system was finally homogenized via the Leeds Jet Homogenizer (University of Leeds, UK), with two passes at $300 \pm 20$ bars. $^{14,27}$ The final concentration of microgels in either the buffer or the two corn syrup solutions was 50.0 vol\%, although the protein content in the microgels was either 10.0 or $15.0 \mathrm{wt} \%$. Volume fraction calculation was based on gravimetric measurement based on previous literature, ${ }^{14}$ however future work should consider calculating volume fraction of the particles based on theoretical models on soft-hairy colloidal spheres. ${ }^{28}$

Preparation of whey protein microgel (WPM) dispersions in the non-Newtonian continuum. Xanthan gum powder ( 0.5 or $1.0 \mathrm{wt} \%$ ) was added to the whey protein microgel dispersion (10.0 wt\% or $15.0 \mathrm{wt} \%$ protein) prepared in buffer (50 vol\% microgels) and stirred at room temperature for $24 \mathrm{~h}$. A final homogenization step was conducted in a water bath at $50{ }^{\circ} \mathrm{C}$ and stirred at $65 \mathrm{rpm}$ for 4 hours. The final concentration of microgels in xanthan gum solutions was 50.0 vol\%, 
Table 1 Sample nomenclature according to the whey protein content in microgels and continuum composition (i.e., xanthan gum or corn syrup content)

\begin{tabular}{llll}
\hline Sample name & $\begin{array}{l}\text { Microgel protein } \\
\text { content (wt\%) }\end{array}$ & $\begin{array}{l}\text { Corn syrup } \\
\text { content (wt\%) }\end{array}$ & $\begin{array}{l}\text { Xanthan gum } \\
\text { content (wt\%) }\end{array}$ \\
\hline Buffer & No particles & 0.0 & 0.0 \\
Buffer10 & 10.0 & 0.0 & 0.0 \\
Buffer15 & 15.0 & 0.0 & 0.0 \\
50CS & No particles & 50.0 & 0.0 \\
50CS10 & 10.0 & 50.0 & 0.0 \\
50CS15 & 15.0 & 50.0 & 0.0 \\
75CS & No particles & 75.0 & 0.0 \\
75CS10 & 10.0 & 75.0 & 0.0 \\
75CS15 & 15.0 & 75.0 & 0.0 \\
0p5XG & No particles & 0.0 & 0.5 \\
0p5XG10 & 10.0 & 0.0 & 0.5 \\
0p5XG15 & 15.0 & 0.0 & 0.5 \\
1XG & No particles & 0.0 & 1.0 \\
1XG10 & 10.0 & 0.0 & 1.0 \\
1XG15 & 15.0 & 0.0 & 1.0 \\
& & &
\end{tabular}

although the protein content in the microgels was either 10.0 or $15.0 \mathrm{wt} \%$.

In order to ease the discussion of results, labels are given to the different samples according to the content of protein in microgels and the type of continuum (i.e., buffer, corn syrup or xanthan gum solution) as described in Table 1.

Dynamic light scattering. The particle size of WPM (10.0 or $15.0 \mathrm{wt} \%$ protein) was measured using a Zetasizer (Nano ZS series, Malvern Instruments, Worcestershire, UK) by means of dynamic light scattering. Colloidal dispersions containing $50.0 \mathrm{vol} \%$ of microgels were diluted in a $20 \mathrm{mM}$ phosphate buffer solution at a 1:500 v/v ratio and placed in a disposable micro plastic cuvette (ZEN 0040). Measurements were performed using a detection angle of $173^{\circ}$. Time dependent correlation functions were fitted using a cumulant analysis satisfying standard criteria to obtain representative particle size distributions. Analysis was performed assuming refractive index of 1.54 and 1.33 for particles and fluid, respectively. The dispersant viscosity was assumed to be the viscosity of water (i.e., $8.9 \times 10^{-4} \mathrm{~Pa} \mathrm{~s}$ ).

Atomic force microscopy (AFM). Whey protein microgels (10.0 or $15.0 \mathrm{wt} \%$ protein) were diluted by a factor of $1000 \mathrm{using}$ $20 \mathrm{mM}$ phosphate buffer solution $(\mathrm{pH}=7)$, deposited $(100 \mu \mathrm{L})$ on clean silicon wafers, and were allowed to adsorb for 10 minutes. Subsequently, to remove the non-adsorbed WPM particles that could adhere onto the AFM tip, the solution was exchanged with $20 \mathrm{mM}$ phosphate buffer using a pipette while ensuring that the sample was kept constantly hydrated. Finally, the samples were transferred to an atomic force microscope (AFM) for imaging.

Topographic images were acquired using a Bruker Multimode 8 AFM equipped with a Bruker Nanoscope $\mathrm{V}$ controller. Silicon nitride AFM cantilevers (model MLCT-DC-BIO) with a nominal spring constant of $0.01 \mathrm{~N} \mathrm{~m}^{-1}$ were purchased from Bruker AFM probes (Camarillo, CA). These cantilevers are thermally stable despite the low spring constant, so it was possible to maintain the force set-point at extremely small values, typically $<100 \mathrm{pN}$ producing the most defined images, whilst at $150 \mathrm{pN}$ sample deformation was clearly observed. Slow line rates of $0.5-0.8 \mathrm{~Hz}$ and gains at the very upper limits of stability were necessary to track the microgels with minimum disturbance. The measurements were performed at room temperature, using a fluid cell loaded with $20 \mathrm{mM}$ phosphate buffer solution $(\mathrm{pH}=7)$. Images were acquired at 768 pixel resolution and processed using Bruker Nanoscope Analysis v1.9. To our knowledge, this is the first study that has carried out AFM imaging of whey protein microgels.

Rheology. All rheological measurements were performed using a Kinexus rheometer (Malvern Instruments Ltd, Worcestershire, UK) equipped with a $60 \mathrm{~mm}$ diameter cone and plate geometry. The experimental temperature was fixed at $37{ }^{\circ} \mathrm{C}$ to mimic oral physiological conditions. The shear viscosity of the continuum and microgel dispersions was obtained for shear rates ranging from 0.1 to $1000.0 \mathrm{~s}^{-1}$. For each point, the measured stress stability was ensured within a $0.5 \%$ of variability. An adiabatic hood was placed in order to reduce the probability of evaporation occurring during the tests. Viscosity curves presented here are obtained as the average of three measurements on freshly prepared samples.

Tribology. Tribological measurements were performed using a Mini Traction Machine (MTM2, PCS instruments, UK) equipped with a tribopair consisting of polydimethylsiloxane (PDMS, Young modulus $\sim 2.6 \mathrm{MPa}$ ) ball-on-disk. A constant load of $2 \mathrm{~N}$ was used in all experiments. Therefore, in this study with the use of a $19.0 \mathrm{~mm}$ diameter ball, the Hertzian pressure corresponded approximately to $200.0 \mathrm{kPa}^{29}$ with a contact radius of $2.0 \mathrm{~mm}$. All measurements were performed at $37{ }^{\circ} \mathrm{C}$ in order to mimic physiological conditions. Friction coefficient $(\mu)$ as a function of the entrainment speed $(U)$ covering a range between 0.001 to $0.3 \mathrm{~m} \mathrm{~s}^{-1}$ with $U=\left(u_{\mathrm{D}}+u_{\mathrm{S}}\right) / 2$, where $u_{\mathrm{D}}$ and $u_{\mathrm{S}}$ were the disc and ball speeds, respectively. Sliding rolling ratio defined as SRR $=\left|u_{\mathrm{D}}+u_{\mathrm{S}}\right| / U$ was kept constant at 0.5. After each measurement, the surfaces were cleaned by 3 cycles of sonication in a $3.0 \mathrm{wt} \%$ solution of Decon 90 for 10 minutes each, followed by 2 cycles of sonication in distilled water. A new set of surfaces was used for different lubricants. Curves of friction coefficients versus entrainment speed presented here are obtained as the average of three measurements on freshly prepared samples. In order to compare the tribological behaviour of microgels dispersions with that of Newtonian fluids, a master curve for the latter is constructed from the Stribeck curves obtained for buffer and corn syrup solutions. For this purpose, the Stribeck curves of the Newtonian fluids with different viscosities are overlapped and an average was found using a smoothing algorithm (i.e., neighbour average of 5 points). The curves are presented like friction as a function of entrainment speed or the product of viscosity and entrainment speed.

Statistics analysis. Significant differences in the experimental data were tested using a one-way ANOVA test with Bonferroni correction. Threshold for significant difference was established at $p<0.05$ using Tukey's Multiple Comparison Test.

\section{Results}

\subsection{Particle size}

Atomic force microscopy (AFM) allowed structural characterisation of the submicron-sized whey protein microgels. The extremely soft 
and adherent nature of the microgels led to large instabilities when using Peak Force Tapping mode (even with its fine and absolute force control) and standard Tapping Mode, hence contact mode was used. Topographical images of the microgels Buffer10 (Fig. 1) deposited on a silicon surface revealed a homogenous distribution of single spherical particles of microgels as well as small clusters that contained between two to six individual particles. The surface of the microgels appeared to be relatively smooth also seen in other polymeric microgels. ${ }^{30}$ Using dynamic light scattering, the average hydrodynamic diameter $\left(D_{\mathrm{h}}\right)$ of the microgels produced at $10.0 \mathrm{wt} \%$ or $15.0 \mathrm{wt} \%$ protein content was estimated to be 86.0 (Fig. 2a) and $92.0 \mathrm{~nm}$ (Fig. 2b), respectively. Such values are in close range of previous protein microgels reported in literature that are designed using top down approach of heat-set crosslinking and controlled shearing. ${ }^{27,31}$

Particle size distribution was also calculated by processing the topographic AFM image with Nanoscope Analysis v1.9 and represented as histograms for microgels containing 10.0 (Fig. 2a) or $15.0 \mathrm{wt} \%$ (Fig. 2b) protein. Size distributions obtained by AFM are in good agreement with DLS measurements regarding

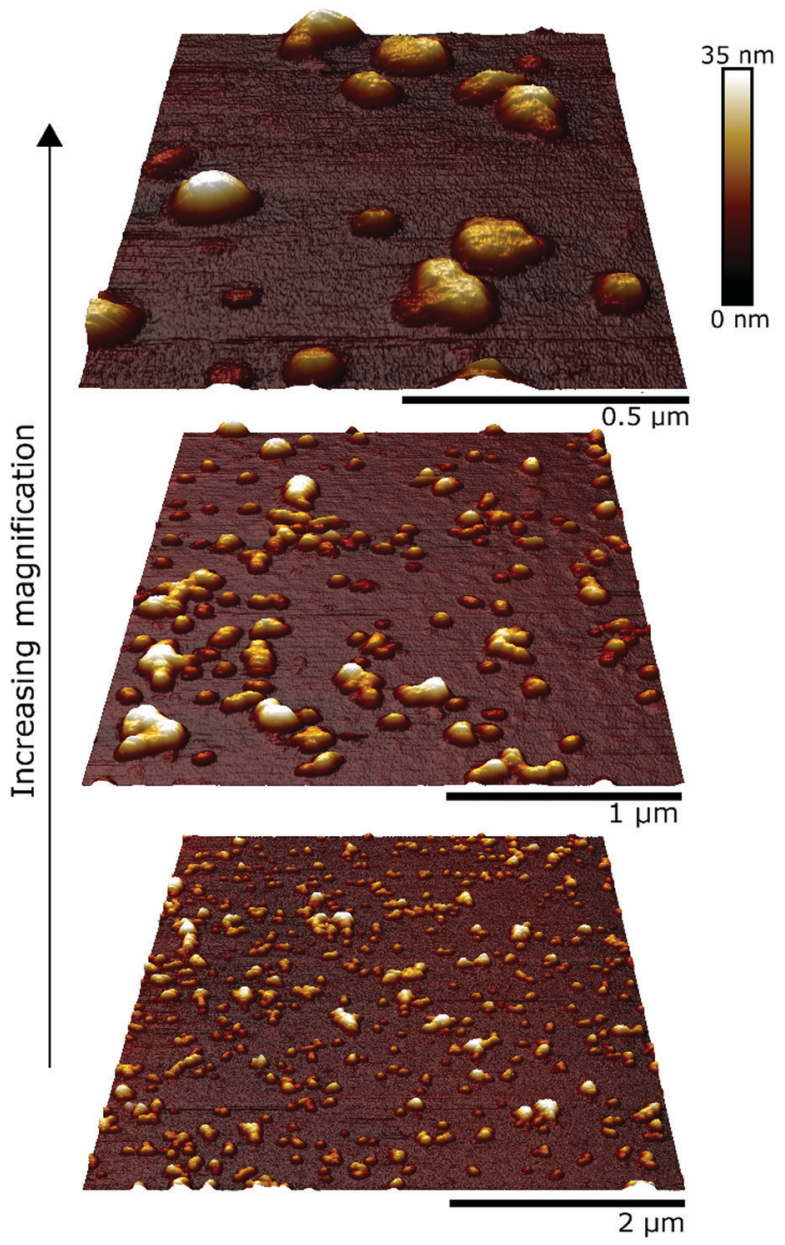

Fig. 1 Three-dimensional topographic images of microgels Buffer10 deposited on silicon substrate, as obtained by contact mode AFM at different magnifications. Most of the particles are distributed homogeneously on the surface, while some of them form aggregates.

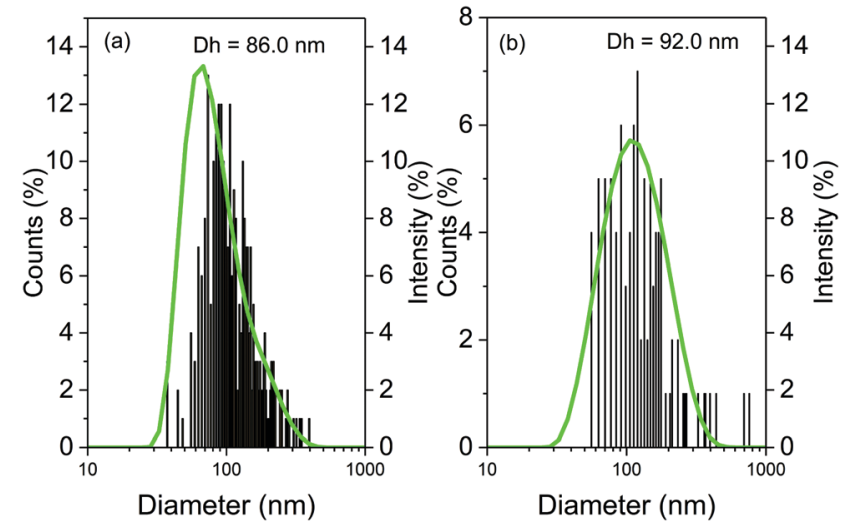

Fig. 2 Histograms showing the particle size distributions obtained by means of atomic force microscopy (AFM) for (a) Buffer10 and (b) Buffer15. Solid green lines show particle size distribution of the same suspensions obtained by means of dynamic light scattering (DLS).

the hydrodynamic radii of the microgels. The particle size distributions of microgels with $10.0 \mathrm{wt} \%$ and $15.0 \mathrm{wt} \%$ protein content dispersed either in buffer or $75.0 \mathrm{wt} \%$ corn syrup solution are presented in the Fig. S1 (ESI $\dagger$ ). The viscosity of the continuum that has been used during microgel formation did not influence the particle size significantly.

\subsection{Rheology}

In this study, microgels were suspended in Newtonian and nonNewtonian fluids, referred as continuum hereafter. Fig. 3 shows the shear viscosity of the different continuums, i.e., phosphate buffer at $\mathrm{pH} 7.0$, corn syrup and xanthan gum solutions without any added microgels. Phosphate buffer (Buffer), $50.0 \mathrm{wt} \%$ (50CS) and $75.0 \mathrm{wt} \%$ (75CS) corn syrup solutions were observed to be Newtonian fluids with viscosities of $0.7,8.0$ and $60.0 \mathrm{mPa}$ s, respectively. Viscosity values found here for corn syrup solution are in agreement with values previously reported in literature. ${ }^{25}$ Xanthan gum solutions are well known shear-thinning fluids, ${ }^{25,32}$

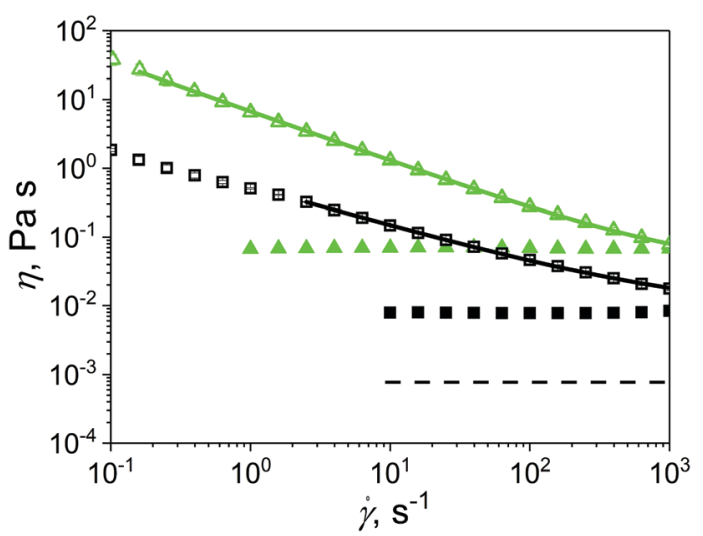

Fig. 3 Shear viscosity of Newtonian and non-Newtonian fluids used as continuum in microgel dispersions. Buffer (dashed line), 50CS (filled square) and $75 \mathrm{CS}$ (filled triangle) are Newtonian fluids with shear viscosity independent to the shear rate $\dot{\gamma}$. Op5XG (open square) and 1XG (open triangle) are shear thinning non-Newtonian fluids. The continuous lines correspond to fittings using eqn (1). 
quality that can be appreciated in Fig. 3 where viscosities of 0p5XG and 1XG decrease at least two orders of magnitude in the shear rate experimental window covering a range from 0.1 to $1000 \mathrm{~s}^{-1}$. At $0.3 \mathrm{~s}^{-1}, 0 \mathrm{p} 5 \mathrm{XG}$ and $1 \mathrm{XG}$ had values of shear viscosity of approximately 1.5 and $30.0 \mathrm{~Pa}$ s, respectively. However, at the highest shear rate $\left(1000 \mathrm{~s}^{-1}\right)$, viscosity values observed for 0p5XG and 1XG approximated the Newtonian viscosities of 50CS and 75CS, respectively. One of the aims of this study was to establish the mechanisms dominating the lubrication capacity of soft colloidal microgel dispersions in complex media using a macroscopic approach. The lubrication performance of some polysaccharide solutions has been successfully explained in terms of hydrodynamics. ${ }^{25}$ In this approach, the strength of hydrodynamic forces are approximated using the fluid viscosity in the high shear rate limit. Hence, in order to obtain an estimate for the high shear rate viscosity plateau $\eta_{\infty}$, experimental data were fitted using the cross model $^{33}$ presented in eqn (1).

$$
\eta(\dot{\gamma})=\frac{\eta_{0}-\eta_{\infty}}{1+(K \dot{\gamma})^{m}}+\eta_{\infty}
$$

Here, $\eta_{0}$ represents zero shear viscosity, $\eta_{\infty}, m$ and $K$ are the fitting parameters. Fittings to shear viscosity curves on xanthan gum experimental data are shown as continuous lines in Fig. 3. Extrapolated values of $\eta_{\infty}$ for xanthan gum solutions 0p5XG and 1XG are $8.0 \mathrm{mPa}$ s and $34.0 \mathrm{mPa}$ s, respectively. As anticipated, the estimated values of $\eta_{\infty}$ were close to the Newtonian viscosities of the corn syrup solutions, Table 2.

In order to appreciate the impact of microgel inclusions on the overall properties of the dispersions, their relative viscosity $\left(\eta_{\mathrm{r}}\right)$ was plotted as function of the Peclet number (Pe) as defined in eqn (2) and (3), respectively.

$$
\begin{gathered}
\eta_{\mathrm{r}}=\frac{\eta}{\eta_{\mathrm{c}}} \\
\mathrm{Pe}=6 \pi \dot{\gamma} \eta_{\mathrm{c}} a^{3} / K_{\mathrm{B}} T
\end{gathered}
$$

where, $\eta$ and $\eta_{\mathrm{c}}$ are the dispersion and continuum viscosities, $a, k_{\mathrm{B}}$ and $T$ are the microgel radius, Boltzmann constant and temperature, respectively. Pe represents the ratio between the characteristic flow time $\left(\dot{\gamma}^{-1}\right)$ and Brownian diffusion time of the microgels. Fig. 4 shows the $\eta_{\mathrm{r}}$ for microgel dispersions in Newtonian continuum. In this representation, dispersions containing microgels with $10.0 \mathrm{wt} \%$ protein content (Buffer10, 50CS10 and 75CS10) show similar shear thinning behaviour, however, this region extends to higher values of $\mathrm{Pe}$ as the particle Brownian diffusion is slowed down on increasing the continuum viscosity. Although influence of particle size is not explored here, in agreement to eqn (3), increasing particle size should also extend the shear thinning region. For instance, shear viscosity reported elsewhere ${ }^{14}$ for dispersions of microgels with diameter of $300.0 \mathrm{~nm}$ (three times larger than reported here) showed a shear thinning region that extended beyond the shear rate (larger than $100.0 \mathrm{~s}^{-1}$ ). In Fig. 4, at the end of the shear thinning region, the second Newtonian plateau is observed, where the values of $\eta_{\mathrm{r}}$ decrease on increasing the continuum viscosity. For instance, microgels in Buffer10 and 50CS10 have an
Table 2 High shear rate viscosity and friction coefficient for $U=0.005 \mathrm{~m} \mathrm{~s}^{-1}$ (boundary/mixed lubrication regime) and mixed $U=0.04 \mathrm{~m} \mathrm{~s}^{-1}$ (mixed lubrication regime) for all microgels studied here

$$
\mu\left(U=0.005 \mathrm{~m} \mathrm{~s}^{-1}, \quad \mu\left(U=0.04 \mathrm{~m} \mathrm{~s}^{-1},\right.\right.
$$

Sample $\eta_{\infty}(\mathrm{Pa})$ boundary*/mixed lubrication) mixed lubrication)

\begin{tabular}{lllc}
\hline Buffer & 0.0007 & $0.67 \pm 0.08^{*}$ & $0.41 \pm 0.06$ \\
Buffer10 & 0.004 & $0.48 \pm 0.1^{*}$ & $0.20 \pm 0.05$ \\
Buffer15 & 0.010 & $0.23 \pm 0.06$ & $0.09 \pm 0.01$ \\
50CS & 0.008 & $0.89 \pm 0.18$ & $0.18 \pm 0.03$ \\
50CS10 & 0.010 & $0.36 \pm 0.09^{*}$ & $0.09 \pm 0.02$ \\
50CS15 & 0.022 & $0.14 \pm 0.07$ & $0.023 \pm 0.005$ \\
75CS & 0.070 & $0.07 \pm 0.05$ & $0.0049 \pm 0.006$ \\
75CS10 & 0.022 & $0.26 \pm 0.05$ & $0.04 \pm 0.02$ \\
75CS15 & 0.041 & $0.07 \pm 0.02$ & $0.009 \pm 0.001$ \\
0p5XG & 0.008 & $0.22 \pm 0.08^{*}$ & $0.09 \pm 0.03$ \\
0p5XG10 & No data & $0.31 \pm 0.04$ & $0.14 \pm 0.03$ \\
0p5XG15 & & $0.12 \pm 0.05$ & $0.05 \pm 0.01$ \\
1XG & 0.034 & $0.08 \pm 0.03$ & $0.020 \pm 0.008$ \\
1XG10 & No data & $0.17 \pm 0.03$ & $0.080 \pm 0.004$ \\
1XG15 & & $0.06 \pm 0.01$ & $0.032 \pm 0.004$
\end{tabular}

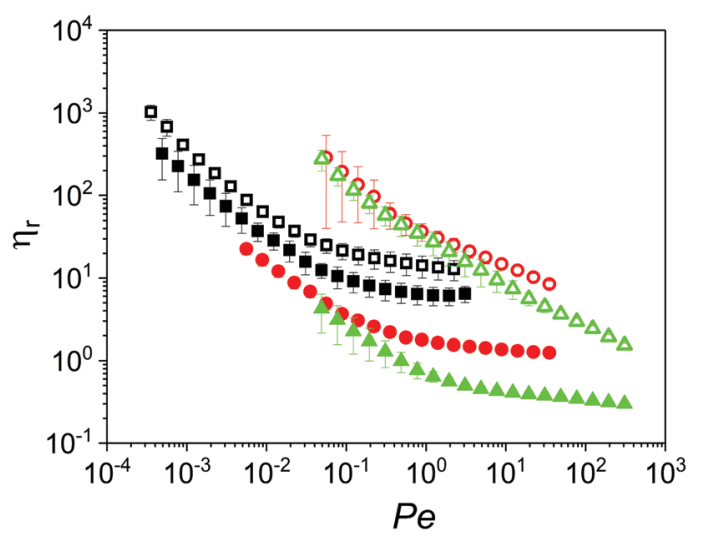

Fig. 4 Relative shear viscosity of microgel dispersions with Newtonian continuum as function of Pe. Buffer10 (close squares), Buffer15 (open squares), 50CS10 (close circles), 50CS15 (open circles), 75CS10 (close triangles) and 75 CS15 (open triangles). The particle volume fraction of microgels is fixed at $50 \mathrm{vol} \%$ in all systems. Error bars represent standard deviations.

incremental effect on the high shear rate viscosity showing values of relative viscosity of about 5.7 and 1.3 , respectively. However, it is clear that increasing continuum viscosity has a detrimental effect on the second plateau viscosity. This is more evident in the case of 75CS10, having the highest viscosity continuum (0.07 $\mathrm{Pa} \mathrm{s})$, where the relative plateau viscosity drops about 0.4 , i.e., absolute viscosity of the microgel dispersion is less than half in comparison to the continuum.

The role of the microgel properties is further explored by increasing their whey protein content to $15.0 \mathrm{wt} \%$. Viscoelasticity of the parent whey protein gels was studied before shearing them into microgels in order to have an estimate of the mechanical properties of microgels. The Fig. S2 (ESI $\dagger$ ) shows the linear elasticity of the parent whey protein gels. The elastic shear modulus of the whey protein gels at $10.0 \mathrm{wt} \%$ and $15.0 \mathrm{wt} \%$ protein content were about 0.1 and $10.0 \mathrm{kPa}$, respectively. Therefore, the microgels containing 10.0 and $15.0 \mathrm{wt} \%$ protein in this study have been referred to as soft and hard microgels, 
respectively. Dispersions of microgels with $15.0 \mathrm{wt} \%$ protein content show differences in the shear thinning region. Sample Buffer15 exhibits similar thinning in comparison to Buffer10 with second Newtonian plateau starting at similar value of $\mathrm{Pe} \sim 0.5$. Interestingly, relative viscosity of Buffer15 shows a twofold increase in comparison to the Buffer10 in the plateau region. The increase in viscosity can be associated to relatively higher elastic modulus of microgels with higher protein content. Here again microgels in Buffer15 appears to act as thickening agents increasing the viscosity relative to the continuum. Samples 50CS15 and 75CS15 show a similar thinning region between them extending towards Pe values higher than the experimental window. However, values of relative viscosity exhibited by these two fluids are considerably higher in comparison to the rest of the experimental fluids in the shear thinning region with $\mathrm{Pe}<2.0$. Thus, their response in the shear thinning region at low Pe numbers splits the microgel dispersions studied here into two groups depending on both, particle rigidity and continuum viscosity. This might be because the definition of Pe does not consider particle deformability during flow. Fitted curves to the experimental data are shown in the Fig. S3 (ESI $\dagger$ ), where absolute viscosity is presented as function of shear rate.

Fig. 5 shows the relative shear viscosity of microgel dispersions in xanthan gum as a function of shear rate. Due to the non-Newtonian nature of the continuum, it is not possible to define a single Brownian diffusion time, thus the use of Pe was not suitable to describe the balance between the hydrodynamic and Brownian relaxation. Fig. 5 shows a detrimental effect of microgel with $10.0 \mathrm{wt} \%$ (0p5XG10 and 1XG10) protein content on the shear viscosity relative to the continuum in the whole range of shear rates studied here. The decrease in viscosity is higher in sample 1XG10 in comparison to 0p5XG10 due to the higher viscosity of the continuum in the former. Both samples started to show an onset of a plateau region at shear rates above $200.0 \mathrm{~s}^{-1}$ with $\eta_{\mathrm{r}}$ about 0.4 and 0.6 for 0 p5XG10 and 1XG10, respectively. Differently, microgels with $15.0 \mathrm{wt} \%$ protein

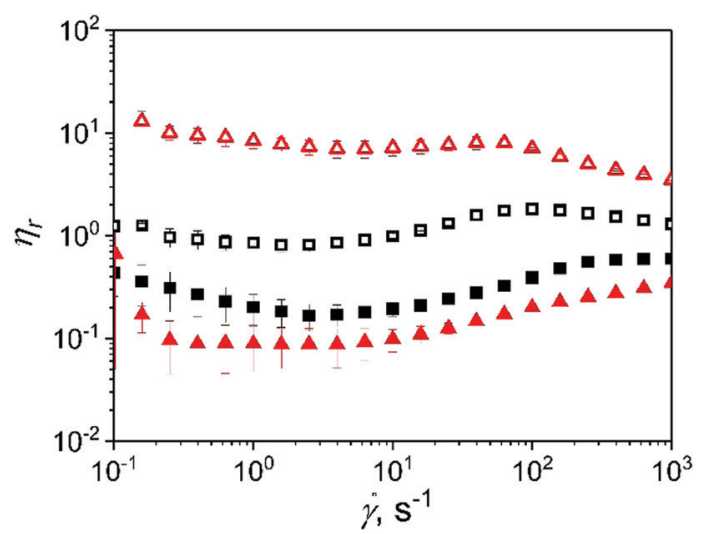

Fig. 5 Relative shear viscosity of microgel dispersions in non-Newtonian continuum. Namely 0p5XG10 (close squares), 0p5XG15 (open squares), 1XG10 (close triangles) and 1XG15 (open triangles). The particle volume fraction of microgels is fixed at 50.0 vol\% in all systems. Error bars represent standard deviations. content exhibit either neutral or active role in the dispersion viscosity depending on the shear conditions and continuum properties.

Relative viscosity of 0p5XG15 is about 1.0 up to shear rates of $1.0 \mathrm{~s}^{-1}$, increasing afterwards to reach value of about 2.0 at $100.0 \mathrm{~s}^{-1}$. Interestingly, 1XG15 shows values of $\eta_{\mathrm{r}}$ as high as 10.0 for shear rates up to $100 \mathrm{~s}^{-1}$. After this point, $\eta_{\mathrm{r}}$ decreases monotonically reaching a value of about 4.0 at the shear rate of $1000.0 \mathrm{~s}^{-1}$. In comparison to the rest of the dispersions, the behaviour of 1 XG15 is counterintuitive since the higher viscosity of the continuum should contribute to decrease $\eta_{\mathrm{r}}$ values. This behaviour is evidence of a synergistic effect between microgels with $15.0 \mathrm{wt} \%$ protein content and the $1.0 \mathrm{wt} \%$ xanthan gum mesh. Further studies are necessary to understand this inversion. In the case of dispersion in xanthan gum continuum, shear viscosity do not show the onset of the second Newtonian plateau making it not possible to fit eqn (1) to the experimental data.

It is important to mention that all rheological discussion has been carried out under the assumption of stick conditions where the macroscopic deformation (imposed at the samplerheometer surface interface) is representative of the deformation at any point of the fluid bulk. However, wall slip is known to be an artefact present when studying highly concentrated dispersions of Brownian solid and soft particles. ${ }^{34}$ Noteworthy, that microgel dispersions studied here exhibit a fluid-like behaviour, where transmission of strain/stress from the rheological surfaces to the fluid bulk is mostly performed through the continuum making the chances of slippage to be highly unlikely. Furthermore, stress curves are shown in the Fig. S4 and S5 (ESI $\dagger$ ) for microgel dispersions in Newtonian and non-Newtonian continuum, respectively. As it can be observed, shear stress increases monotonically showing no signs of discontinuities suggesting no obvious wall slippage. Overall, the rheological analyses are intended to provide an explanation to the tribological performance of the microgels presented in the next section.

\subsection{Soft tribology}

Dispersions of whey protein microgels in aqueous media have demonstrated the capability to reduce friction in soft contacts. For example, Sarkar et al. studied the lubrication properties of $300 \mathrm{~nm}$ diameter whey protein $(10.0 \mathrm{wt} \%)$ microgels dispersed in aqueous media. ${ }^{14}$ They demonstrated the capability of colloidal whey protein microgels to reduce friction in compliant sliding/rolling contacts. In this study, the microgels were threetimes smaller (Fig. 1 and 2) and also prepared with different protein content resulting in different degree of protein crosslinking and elasticity (Fig. S2, ESI $\dagger$ ) and thus expected to have different lubrication performance in absence or presence of the complex continuum. Fig. 6 shows the lubrication performance of microgel dispersions in comparison to their respective continuum (black squares) in the form of Stribeck curves, where the friction coefficients are shown as a function of entrainment speed. Stribeck curves in Fig. 6a show the boundary and mixed lubrication regimes. The boundary regime is identified as the region where friction coefficient is independent of the 
entrainment speed. On the other hand, in the mixed lubrication regime, the friction coefficient decreases monotonically on increasing the speed. The presence of microgels helped decreasing the contact friction in the mixed lubrication regime, in comparison to the continuous phase alone (Buffer), however no significant differences are found in the boundary region. For example, at entrainment speed $U=0.005 \mathrm{~m} \mathrm{~s}^{-1}$ corresponding to the boundary regime, friction coefficient show no significant difference between Buffer and Buffer10 $(p>0.05)$. At the same speed, sample Buffer15 was found to be performing in the mixed lubrication regime as evident in the decrease of friction coefficient on increasing entrainment speed. Thus, at $U=0.005 \mathrm{~m} \mathrm{~s}^{-1}$, friction coefficient for Buffer15 was about 0.23, significantly lower $(p<0.05)$ than values obtained for Buffer and Buffer10. At $U=0.04 \mathrm{~m} \mathrm{~s}^{-1}$, Buffer, Buffer10 and Buffer15 were found to be working in the mixed lubrication regime. Friction coefficient of buffer was about three to five times higher with respect to the friction coefficient values for Buffer10 and Buffer15, respectively $(p<0.05)$. These observations show the influence of the microgel mechanical properties (i.e., elastic modulus) on their lubrication performance, the higher the particle elastic modulus (i.e., higher protein content) the lower was the contact friction, at least in the mixed lubrication regime. Friction coefficient Buffer10 is similar to friction measurements (using the same set-up) reported elsewhere ${ }^{14}$ for similar whey protein microgels $(10.0 \mathrm{wt} \%)$ with average diameter three times larger. Thus, increasing particle size of the microgels might have no influence on the tribological performance of the final dispersion. A systematic study on impact of average microgel size on lubrication properties is required; however, this is out of scope of the present study. All values of friction are summarised in Table 2 with the corresponding values of $\eta_{\infty}$ obtained using eqn (1) except for microgels in xanthan gum solutions.

Fig. 6c presents the Stribeck curves obtained for microgels in $50 \mathrm{wt} \%$ syrup solutions (50CS10 and 50CS15) as well as the continuum alone (50CS). Stribeck curves for 50CS and 50CS10 showed the boundary and mixed lubrication regimes. Sample 50CS15 showed the mixed lubrication regime and the onset of the elastohydrodynamic lubrication regime. The latter is observed as an upturn of the Stribeck curve with friction coefficients increasing with the entrainment speeds above $0.1 \mathrm{~m} \mathrm{~s}^{-1}$. At $U=0.005 \mathrm{~m} \mathrm{~s}^{-1}$, friction coefficient obtained for $50 \mathrm{CS} 10$ is about 0.36 , twice as low in comparison to CS50 (plain continuum) at the same entrainment speed. While at $U=0.005 \mathrm{~m} \mathrm{~s}^{-1}, 50 \mathrm{CS}$ is found to be working in the boundary regime (friction coefficient independent of speed), 50CS10 is working in the mixed lubrication regime showing a subtle decrease in friction on increasing entrainment speed. 50CS75 is also found to be working in the mixed lubrication regime with friction coefficient about 0.14 , approximately four and two times lower in comparison to 50CS and 50CS10, respectively. This confirms the positive influence of increasing whey protein in microgels, decreasing the friction coefficient as observed also for microgels dispersed in buffer (Fig. 6a). In Fig. 6b, at entrainment speed $U=0.04 \mathrm{~m} \mathrm{~s}^{-1}, 50 \mathrm{CS}, 50 \mathrm{CS} 10$ and 50CS15
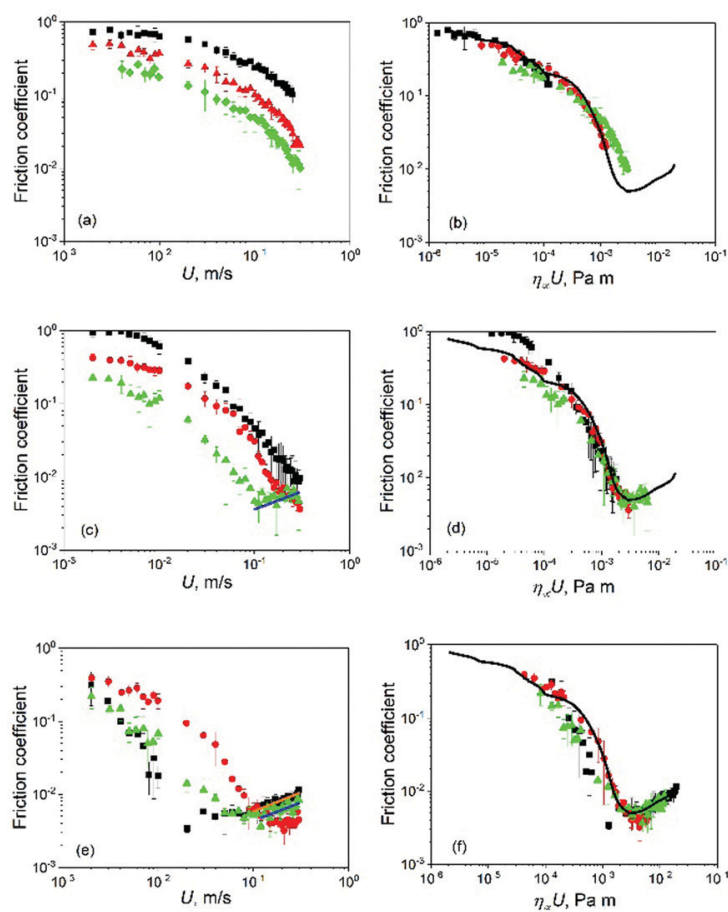

Fig. 6 Friction coefficient as a function of $U$ (a, c and e) or $\eta_{\infty} U$ (b, d and f) for microgel dispersions in Newtonian continuum. (a and b) Buffer (squares), Buffer10 (circles) and Buffer15 (triangles). (c and d) 50CS (squares), 50CS10 (circles) and 50CS15 (triangles). (e and f) 75CS (squares), $75 \mathrm{CS} 10$ (circles) and 75CS15 (triangles). The particle volume fraction of microgels is fixed at 50.0 vol\% in all systems. Continuous line in b, d and e represents an average Stribeck curve of Newtonian fluids (buffer and corn syrups) used in this study. Continuous lines in $c$ and $d$ are fittings to the hydrodynamic lubrication regime using eqn (4). Error bars represent standard deviations.

performed in the mixed lubrication regime. While values of friction coefficients for 50CS and 50CS10 are not significantly different $(p>0.05)$, the friction coefficient measured for 50CS15 was significantly lower in comparison to both, 50CS and 50CS10. Moreover, at approximately $U=0.1 \mathrm{~m} \mathrm{~s}^{-1}$, the onset of the hydrodynamic lubrication can be clearly appreciated only in the curve corresponding to 50CS15.

In the case of simple Newtonian fluids, increasing lubricant viscosity decreases the speed at which the elastohydrodynamic lubrication regime starts. ${ }^{24}$ This can be clearly observed comparing 50CS (Fig. 6c) and 75CS (Fig. 6e), both Newtonian fluids having viscosities of 0.008 and $0.07 \mathrm{~Pa} \mathrm{~s}$, respectively. Due to its larger viscosity, 75CS displays the elastohydrodynamic lubrication, while in the same range of speeds 50CS shows only the mixed regime. Applying this reasoning to dispersions 50CS10 and 50CS15 in Fig. 6c, the appearance of an elastohydrodynamic regime for the latter indicates larger viscosity in comparison to the former.

In Fig. 6e, Stribeck curves correspond to 75CS and microgels dispersions 75CS10 and 75CS15. Opposite to the observations in dispersion in lower viscosity continuum (i.e., Buffer10, 50CS10), microgels in 75CS10 increased friction in the mixed lubrication regime in comparison to the continuum alone 
(75CS). For instance, at $U=0.005 \mathrm{~m} \mathrm{~s}^{-1}$, friction coefficient found for $75 \mathrm{CS} 10$ is about 0.26 , representing more than a threefold increase with respect to 75CS. 75CS and 75CS15 show similar lubrication, however the onset of elastohydrodynamic lubrication reveals differences in viscosity. Onset of elastohydrodynamic lubrication of 75CS appears at the lowest speed at about $0.03 \mathrm{~m} \mathrm{~s}^{-1}$, followed by $75 \mathrm{CS} 15$ at $0.07 \mathrm{~m} \mathrm{~s}^{-1}$ and finally 75CS10 shows the end of mixed lubrication (and start of elastohydrodynamic regime) at a higher speed of about $0.2 \mathrm{~m} \mathrm{~s}^{-1}$. Thus, in agreement to discussion presented above, viscosity values of the lubricants follow the same order from highest to lowest, i.e., 75CS $>75 \mathrm{CS} 15>75 \mathrm{CS} 10$. This is also in line with values of viscosity obtained by means of steady shear rheological experiments as shown in Table 2 . The appearance of the hydrodynamic lubrication regime in friction curves for samples 50CS15, 75CS and 75CS15 makes it possible to have an estimation of effective viscosity from a tribological perspective. de Vicente et al., ${ }^{35}$ provided an arithmetic expression for the soft-hydrodynamic lubrication regime of a rolling/sliding ball on plate contact as introduced in eqn (4):

$$
\mu=1.46 \bar{U}^{0.65} \bar{W}^{-0.70}+\operatorname{SRR}\left(3.8 \bar{U}^{0.71} \bar{W}^{-0.76}+0.96 \bar{U}^{0.36} \bar{W}^{-0.11}\right)
$$

Here, SRR is the sliding/rolling ratio, taking values from 0.0 in pure rolling conditions to 2.0 in the pure sliding conditions. In eqn (4), $\bar{U}=\frac{U \eta}{E^{*} R^{\prime}}$ and $\bar{W}=\frac{W}{E^{*} R^{\prime 2}}$, where $E^{\prime}$ and $R^{\prime}$ are the reduced Young modulus and the ball radius, respectively. Eqn (4) was successfully fitted to the experimental data using viscosity as the only fitting parameter. Fittings are presented as lines in Fig. 6. Values of viscosity estimated for 50CS15, 75CS and 75CS15 using eqn (4) are 0.017, 0.05 and $0.025 \mathrm{~Pa} \mathrm{~s}$, respectively. Although in comparisons to values presented in Table 2, values obtained using eqn (4) are slightly lower, the trend obtained in rheology is replicated. Thus, when dispersed in the highest viscosity continuum CS75 (0.07 Pa s), microgels have a detrimental effect on both, rheological and lubrication properties.

A common procedure to compare the tribological performance of different fluids is accomplished by using their rheological properties representing friction coefficient as function of the product of viscosity and entrainment speed. In the case of Newtonian fluids, this procedure is well known to deliver master curves (overlapping of curves representing different lubricants) that covers at least the mixed and hydrodynamic lubrication regimes, where fluid dynamics plays an important role in the lubrication. ${ }^{35}$ Although for most nonNewtonian lubricants the viscosity is a function of working conditions (i.e., shear rate), a similar representation is commonly found where the Newtonian viscosity is replaced by a value of viscosity at a high shear rate (above $1000.0 \mathrm{~s}^{-1}$ ). ${ }^{25}$ This approximation relies on the fact that the tribological limit imposes high shear rates on the lubricant due to the proximity between contact surfaces involving submicron separations. Here, the relation between lubrication performance of non-Newtonian microgel dispersions and their rheology is carried out by representing the friction coefficient as function of the product of entrainment speed and $\eta_{\infty}$. In fact, the successful fitting of eqn (4) in the elastohydrodynamic lubrication regime is a clear indication that this procedure is adequate at least for microgels in Newtonian continuum. In the case of lubricants 75CS10 and $75 \mathrm{CS} 15$, the plateau was not observed in the experimental window and values for $\eta_{\infty}$ were estimated by extrapolation using eqn (1). Fig. $6 \mathrm{~b}$, d and e show friction coefficient as function of the product $U \cdot \eta_{\infty}$ for microgel dispersion with Buffer, 50CS and 75CS as continuum, respectively. A Stribeck curve representing the average lubrication performance of Newtonian lubricants is also presented for comparison purposes. There is a reasonable overlap of Stribeck curves for dispersions regardless of their continuum and protein content in the microgels. Hence, lubrication performance of colloidal microgel dispersions in media with different viscosity (covering three orders of magnitude) can be approximated by considering them as Newtonian fluids having viscosity $\eta_{\infty}$. In other words, tribological deformation corresponds to the rheological shear rates in the second Newtonian plateau of the microgel dispersions, which has been seldom reported in literature. It is worth noticing that lubricants 50CS15 in Fig. 6d, 75CS10 and 75CS15 in Fig. 6f, show a clear overlap in the onset of the transition between the mixed and hydrodynamic lubrication regime. This is an important indication that hydrodynamic forces acting on the microgel dispersions in the soft tribological limit are still dominated by the rheological response in the equivalent shear rate range, i.e., high shear rate limit.

Having established the influence of microgels in Newtonian continuum of different viscosities in the rheological and tribological limit, a similar study was also conducted for the nonNewtonian continuum, i.e., xanthan gum solutions. Fig. 7a shows the Stribeck curves obtained for microgels dispersions 0p5XG10 and 0p5XG15 in comparison to their continuum alone (0p5XG). Stribeck curves for all lubricants shown in Fig. 7a show only the mixed lubrication regime. In Fig. 7a, only 0p5XG15 shows significant differences. For instance, at entrainment speed 0.005 and $0.04 \mathrm{~m} \mathrm{~s}^{-1}$, friction coefficient values for 0p5XG15 are about half the values found for 0p5XG and 0p5XG10 at the same speeds. Fig. 7b shows the Stribeck curves for microgels dispersed in the highest viscosity nonNewtonian continuum (1XG). While Stribeck curves for 1XG and 1XG15 show no relative differences, friction coefficients obtained for 1XG10 are significantly higher in comparison to the other two curves up to $U=0.1 \mathrm{~m} \mathrm{~s}^{-1}$, where a minimum for the friction coefficient is reached. The appearance of a hydrodynamic lubrication regime is only evident in the case of 1XG15, where fitting of eqn 4 generates a value of viscosity of about 0.2 Pa s. Using this calculation, a comparison between rheological and tribological performance can be carried out for $1 \mathrm{XG}$ and 1XG15. Effective viscosity of 1XG15 in the elastohydrodynamic regime (eqn (4)) represents a six-fold increase in comparison to the high shear viscosity value extrapolated for 1XG (Table 2). However, in the mixed lubrication regime, no significant differences are found between friction coefficients of $1 \mathrm{XG}$ and 1XG15. Thus, it is clear that a single value of 

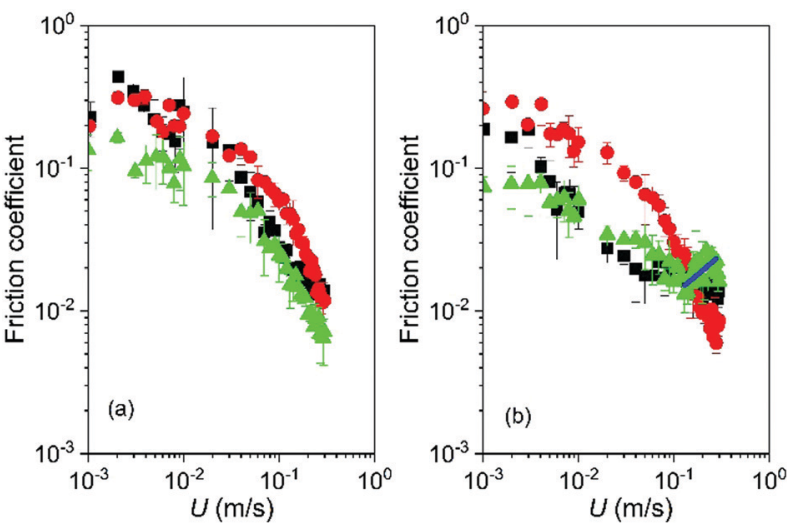

Fig. 7 Friction coefficient as a function of $U$ for the microgel dispersions non-Newtonian continuum. (a) 0p5XG (squares), 0p5XG10 (circles) and 0p5XG15 (triangles). (b) 1XG (squares), 1XG10 (circles) and 1XG15 (triangles). The particle volume fraction of microgels is fixed at 50 vol\% in all fluids. Continuous lines in $\mathrm{b}$ represent fitting to the hydrodynamic lubrication regime using eqn (4). Error bars represent standard deviations.

viscosity $\eta_{\infty}$ cannot be used to explain the lubrication performance of microgels in non-Newtonian continuum (xanthan gum). In addition, in the non-Newtonian continuum, microgels in the lowest viscosity continuum (0p5XG) can improve the lubrication with respect to the continuum and the same microgels can cause a detrimental effect when dispersed in the higher viscosity continuum (1XG). Regardless of the continuum properties, lubrication performance of the microgel dispersion is better for the higher protein content particles.

\section{Discussion}

Fig. 8 summarizes the role of microgels on the high shear rheology and lubrication performance of all the microgel dispersions studied. To do so, the relative friction coefficient,

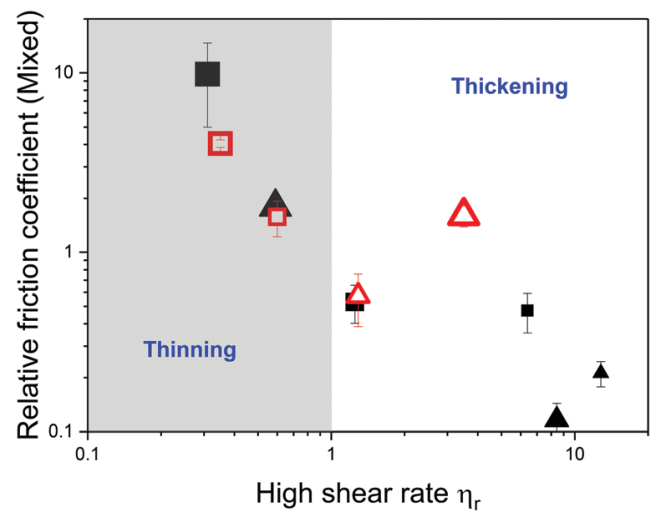

Fig. 8 Relative friction coefficient as function of high shear rate relative viscosity for all fluids studied here. Square and triangle shaped symbols represent microgels with 10.0 and $15.0 \mathrm{wt} \%$ protein content, respectively. Relative friction coefficients were calculated using absolute values of friction coefficient corresponding to that of the mixed lubrication regime values obtained at $U=0.04 \mathrm{~m} \mathrm{~s}^{-1}$. Closed symbols correspond to Newtonian continuum, whilst open symbols are for non-Newtonian continuum. Increasing symbol size represent higher continuum viscosity. defined as the ratio of the friction coefficient of the dispersions to the value of the plain continuum, is represented as function relative viscosity in the high shear rate limit. Friction coefficient values used here correspond to an entrainment speed of $0.04 \mathrm{~m} \mathrm{~s}^{-1}$, which lies in the mixed lubrication regime for all dispersions. Choice of relative viscosity differs between dispersions in Newtonian and non-Newtonian continuum. For dispersions in buffer or corn syrup solutions, the viscosity corresponds to the extrapolated values $\eta_{\infty}$ using eqn (1). Since extrapolation was not suitable for dispersions in non-Newtonian continuum, viscosity used in Fig. 8 corresponds to values at the highest shear rate accessible $\left(1000.0 \mathrm{~s}^{-1}\right)$. It is evident that lubrication and high shear rate viscosity are closely related, with friction coefficients decreasing as the dispersion viscosity increases for both, Newtonian and non-Newtonian continuum.

Using the definition of relative viscosity, data presented in Fig. 8 is divided in two regions representing the influence of microgels as thickening or thinning agents. In the thinning region, relative high shear rate viscosity is lower than 1.0, with microgels decreasing the viscosity with respect to the continuum. Whether a dispersions falls in either regime depends on both, continuum viscosity and the mechanical properties of the microgel particles.

This is true for both, Newtonian and non-Newtonian continuum. Based on a balance between stored (in soft elastic particles) and dissipated (by Newtonian continuum) energy during shear flow, Avazmohammadi and Castaneda ${ }^{36}$ estimated the viscosity of soft particles dispersions in Newtonian continuum as function of the dimensionless parameter $\mathrm{Ca}$ defined in eqn (5), as shown below:

$$
\mathrm{Ca}=\dot{\gamma} \eta_{\mathrm{c}} / G
$$

with $G$ as the particle elastic modulus. They demonstrated that a value of $\mathrm{Ca}=0.88$ represented a threshold for the rheological performance of dispersions of soft elastic particles. Below this value, relative viscosity of the dispersion was greater than 1.0 regardless of particle concentration, however, above this value, relative viscosity drops below 1.0. In order to understand the role of particle elasticity on the rheological response of our microgel dispersions, eqn (5) is evaluated using the elasticity of the parent gels as estimates for the mechanical properties of microgels in Newtonian continuum. The highest value for Ca obtained for a dispersion in the thinning region of Fig. 8 is 0.07 corresponding to $75 \mathrm{CS} 10$ when eqn (5) is evaluated at a shear rate of $100.0 \mathrm{~s}^{-1}$. This value of shear rate is chosen since it is the starting of the second Newtonian plateau for 75CS10 as shown in Fig. S3 (ESI $\dagger$ ). The fact that $\mathrm{Ca}$ value calculated for 75CS10 is an order of magnitude lower than the threshold value found by Avazmohammadi and Castaneda ${ }^{36}(\mathrm{Ca}=0.88)$ indicates that these soft microgels with low elastic modulus (high deformability) are not solely responsible for their behaviour as thinning agents. An alternative explanation for relative viscosity lower than one is draining of the buffer from particles into the continuum decreasing the effective concentration of the corn syrup, hence decreasing the effective viscosity of the continuum and dispersion. The use of eqn (5) is restricted to 
Table 3 Film thickness $h_{\mathrm{c}}$ and shear rates calculated for dispersions showing elastohydrodynamic lubrication regime in tribological experiments. Values of viscosity and entrainment speed used for calculations are also shown

\begin{tabular}{lllll}
\hline Dispersion & Viscosity $(\mathrm{Pa})$ & $U\left(\mathrm{~m} \mathrm{~s}^{-1}\right)$ & $h_{\mathrm{c}}(\mathrm{m})$ & Shear rate $\left(\mathrm{s}^{-1}\right)$ \\
\hline 50CS10 & 0.017 & 0.10 & $7.4 \times 10^{-6}$ & 6764.0 \\
75CS & 0.050 & 0.03 & $6.9 \times 10^{-6}$ & 2187.0 \\
75CS15 & 0.025 & 0.07 & $7.5 \times 10^{-6}$ & 4653.0 \\
1XG15 & 0.200 & 0.10 & $3.2 \times 10^{-5}$ & 1541.0
\end{tabular}

Newtonian continuum and thus not suitable to describe microgels in xanthan gum solutions. However, since high shear rate viscosity of Newtonian and non-Newtonian continuums is similar, it can be argued that even in the case of microgels in xanthan gum solutions, low elastic modulus of microgels is not responsible for the drop in relative viscosity below 1.0. Since the hydrodynamic-elastic balance is not capable to explain the reduction of viscosity, other mechanisms possible include particle draining and disruption of the xanthan gum network by microgels due to possible polymer-particle interactions.

Finally, a rough estimation of the shear rates in the elastohydrodynamic lubrication regime is possible by using the expression provided by de Vicente $e t$ al. ${ }^{35}$ for the central film thickness as shown in eqn (6).

$$
h_{\mathrm{c}}=3.3 R^{\prime} \bar{U}^{0.6} \bar{W}^{-0.14}
$$

The shear rate is then approximated as $\dot{\gamma}=\mathrm{SRRU} / h_{\mathrm{c}}$. Samples showing elastohydrodynamic regime in the range of entrainment speeds studied here are 50CS15, 75CS, 75CS15 and 1XG15. Table 3 shows estimation of central film thickness and shear rate together with values of viscosity and speed used to evaluate eqn (6). Entrainment speed values used for calculations correspond to the onset of elastohydrodynamic lubrication. Viscosity corresponds to values obtained from fitting eqn (4) to the elastohydrodynamic lubrication regime of the different dispersions. Values of $h_{\mathrm{c}}$ for all microgels in Newtonian continuum are about $7.0 \times 10^{-6} \mathrm{~m}$, whilst for dispersion in xanthan gum solution thickness is one order of magnitude higher i.e., about $3.0 \times 10^{-5} \mathrm{~m}$. Nevertheless, in all cases, shear rate is above $1000.0 \mathrm{~s}^{-1}$. This provides further justification to the use of second Newtonian plateau viscosity to represent the tribological performance of microgels in Newtonian continuum. In the case of microgels in non-Newtonian continuum (xanthan gum solution), their tribological performance is not simply represented by one viscosity value. However, their elastohydrodynamic lubrication regime might be well approximated by their bulk viscosity.

\section{Conclusions}

Soft whey protein microgels with average size of about $100.0 \mathrm{~nm}$ (determined by AFM and DLS) dispersed in Newtonian and non-Newtonian continuum were investigated by means of rheology and soft tribology. Steady shear rheology and soft tribology experiments showed that the lubrication performance of microgels dispersed in either Newtonian or non-Newtonian continuum (xanthan gum solution) is related to their hydrodynamics. On the one hand, the tribological performance of microgels dispersed in Newtonian continuum can be quantitatively explained in terms of their second Newtonian plateau bulk viscosity. On the other hand, for microgels dispersed in xanthan gum solutions, the relation between bulk rheology and soft tribological performance is qualitative.

Microgels enhanced the rheological and tribological performance when dispersed in continuum with relatively low viscosity, increasing the viscosity i.e., acting as thickeners, thus lowering the friction coefficient. However in high viscosity continuum, microgels caused a detrimental effect in mechanical performance, decreasing the viscosity and increasing the friction coefficients. Regardless of being dispersed in Newtonian or non-Newtonian continuum, increasing the elastic modulus of the microgels (higher protein content) showed benefits by increasing the high shear rate viscosity of the final dispersion. This consequently improved their tribological performance, i.e., decreased the friction coefficients. Thus, in order to obtain a benefit from microgels on the tribology performance of a dispersion, it is necessary to have harder particles when the continuum viscosity is increased. However, further studies are necessary to determine when microgels become too hard that they can increase the abrasiveness in biological contacts. Future work should look into a more thorough calculation of the effective volume fraction of the soft microgel particles in order to compare with theoretical models.

\section{Conflicts of interest}

The authors declare no conflict of interest.

\section{Acknowledgements}

This study has received funding from the European Research Council (ERC) under the European Union's Horizon 2020 research and innovation programme (grant agreement no 757993).

\section{References}

1 J. K. Oh, R. Drumright, D. J. Siegwart and K. Matyjaszewski, Prog. Polym. Sci., 2008, 33, 448-477.

2 N. Murthy, M. C. Xu, S. Schuck, J. Kunisawa, N. Shastri and J. M. J. Frechet, Proc. Natl. Acad. Sci. U. S. A., 2003, 100, 4995-5000.

3 S. V. Vinogradov, Curr. Pharm. Des., 2006, 12, 4703-4712.

4 A. Sarkar, S. N. Zhang, M. Holmes and R. Ettelaie, Adv. Colloid Interface Sci., 2019, 263, 195-211.

5 G. Q. Liu, Z. L. Liu, N. Li, X. L. Wang, F. Zhou and W. M. Liu, ACS Appl. Mater. Interfaces, 2014, 6, 20452-20463.

6 A. Sarkar, B. Murray, M. Holmes, R. Ettelaie, A. Abdalla and X. Y. Yang, Soft Matter, 2016, 12, 3558-3569. 
7 O. Sterner, C. Karageorgaki, M. Zurcher, S. Zurcher, C. W. Scales, Z. Fadli, N. D. Spencer and S. G. P. Tosatti, ACS Appl. Mater. Interfaces, 2017, 9, 20150-20160.

8 E. M. Krop, M. M. Hetherington, M. Holmes, S. Miquel and A. Sarkar, Food Hydrocolloids, 2019, 88, 101-113.

9 E. M. Krop, M. M. Hetherington, S. Miquel and A. Sarkar, Food Qual. Prefer., 2019, 74, 118-124.

10 S. Jahn and J. Klein, Macromolecules, 2015, 48, 5059-5075.

11 M. Wathier, B. A. Lakin, P. N. Bansal, S. S. Stoddart, B. D. Snyder and M. W. Grinstaff, J. Am. Chem. Soc., 2013, 135, 4930-4933.

12 G. Q. Liu, X. L. Wang, F. Zhou and W. M. Liu, ACS Appl. Mater. Interfaces, 2013, 5, 10842-10852.

13 O. Torres, E. Andablo-Reyes, B. S. Murray and A. Sarkar, ACS Appl. Mater. Interfaces, 2018, 10, 26893-26905.

14 A. Sarkar, F. Kanti, A. Gulotta, B. S. Murray and S. Y. Zhang, Langmuir, 2017, 33, 14699-14708.

15 M. L. Olivares, K. Shahrivar and J. de Vicente, J. Food Eng., 2019, 245, 157-165.

16 I. F. Farres, M. Douaire and I. T. Norton, Food Hydrocolloids, 2013, 32, 115-122.

17 A. Gabriele, F. Spyropoulos and I. T. Norton, Soft Matter, 2010, 6, 4205-4213.

18 D. A. Garrec and I. T. Norton, Food Hydrocolloids, 2013, 33, 160-167.

19 J. de Vicente, H. A. Spikes and J. R. Stokes, J. Tribol., 2006, 128, 795-800.

20 I. F. Farres and I. T. Norton, Food Hydrocolloids, 2015, 45, 186-195.

21 T. Kairn, P. J. Daivis, I. Ivanov and S. N. Bhattacharya, J. Chem. Phys., 2005, 123, 194905.
22 R. G. Larson and P. S. Desai, Annu. Rev. Fluid Mech., 2015, 47, 47-65.

23 S. Adams, W. J. Frith and J. R. Stokes, J. Rheol., 2004, 48, 1195-1213.

24 J. de Vicente, J. R. Stokes and H. A. Spikes, Proc. Inst. Mech. Eng., Part J, 2006, 220, 55-63.

25 J. de Vicente, J. R. Stokes and H. A. Spikes, Food Hydrocolloids, 2006, 20, 483-491.

26 J. R. Stokes, L. Macakova, A. Chojnicka-Paszun, C. G. de Kruif and H. H. J. de Jongh, Langmuir, 2011, 27, 3474-3484.

27 A. Araiza-Calahorra and A. Sarkar, Food Struct., 2019, 21, 100113.

28 W. C. K. Poon, E. R. Weeks and C. P. Royall, Soft Matter, 2012, 8, 21-30.

29 A. Sarkar, E. Andablo-Reyes, M. Bryant, D. Dowson and A. Neville, Curr. Opin. Colloid Interface Sci., 2019, 39, 61-75.

30 A. Aufderhorst-Roberts, D. Baker, R. J. Foster, O. Cayre, J. Mattsson and S. D. Connell, Nanoscale, 2018, 10, 16050-16061.

31 A. Sarkar, V. Ademuyiwa, S. Stubley, N. H. Esa, F. M. Goycoolea, X. F. Qin, F. Gonzalez and C. Olvera, Food Hydrocolloids, 2018, 84, 282-291.

32 O. Torres, A. Yamadaa, N. M. Rigby, T. Hanawab, Y. Kawano and A. Sarkar, Biotribology, 2019, 17, 8-18.

33 J. Xie and Y. C. Jin, Eng. Appl. Comp. Fluid, 2016, 10, 111-129.

34 S. P. Meeker, R. T. Bonnecaze and M. Cloitre, J. Rheol., 2004, 48, 1295-1320.

35 J. de Vicente, J. R. Stokes and H. A. Spikes, Tribol. Lett., 2005, 20, 273-286.

36 R. Avazmohammadi and P. P. Castaneda, J. Fluid Mech., 2015, 763, 386-432. 